\title{
Correction to: Nonlinear, Nonlocal and Fractional Turbulence
}

\section{Correction to:}

\section{P. W. Egolf, K. Hutter,}

Nonlinear, Nonlocal and Fractional Turbulence, https://doi.org/10.1007/978-3-030-26033-0

Below corrections are updated in book.

Text book cover image,

third line from bottom: ... with permission. (C2019 American Institute of Physics

Page $v, 2^{\text {nd }}$ line: ... of irregularity, with all its facets, is a main topic...

$5^{\text {th }}$ line: ..., the main ingredients in life, art, music, etc.

$7^{\text {th }}$ line $\ldots$ order and disorder, with different

$\mathbf{8}^{\text {th }}$ line ...tempi and intensities, gives our...

$11^{\text {th }}$ line ... (energy input), it gives...

$\mathbf{2 0}^{\text {th }}$ line ...(from its big bang), but even the creation...

Page vi, $17^{\text {th }}$ line $\mathrm{He}$, at ... \footnotetext{
https://doi.org/10.1007/978-3-030-26033-0_1 https://doi.org/10.1007/978-3-030-26033-0_2 https://doi.org/10.1007/978-3-030-26033-0_3 https://doi.org/10.1007/978-3-030-26033-0_5 https://doi.org/10.1007/978-3-030-26033-0_6 https://doi.org/10.1007/978-3-030-26033-0_7 https://doi.org/10.1007/978-3-030-26033-0_8 https://doi.org/10.1007/978-3-030-26033-0_9 https://doi.org/10.1007/978-3-030-26033-0_10 https://doi.org/10.1007/978-3-030-26033-0_11 https://doi.org/10.1007/978-3-030-26033-0_12 https://doi.org/10.1007/978-3-030-26033-0
}

The updated online versions of the chapters can be found at 
$\mathbf{1 8}^{\text {th }}$ line $\ldots$. his time, did not distinguish between art and science.

Page vii, Pic. 3, Tab. Capt.: In June 1889, the famous impressionist artist Vincent van Gogh painted the picture "starry night" by oil on canvas.

$\mathbf{1}^{\text {st }}$ line: In the literature on turbulence, Leonardo da Vinci's drawings. . .

Page $\mathbf{x}, \mathbf{3}^{\text {rd }}$ line: This is all well known, as is the fact...

$16^{\text {th }}$ line: ... a flux law, in analogy to Fick's law...

Page xi, $5^{\text {th }}$ line: In April 1985, one of us (Peter William Egolf - PWE), deriving the Difference-Quotient Turbulence Model (DQTM), got automatically involved into the problem of nonlocality and started to tackle it.

$1^{\text {th }}$ line: ... engineering experts, applying often stronger...

$16^{\text {th }}$ line: ... and, thereby, describes a hereditary...

$25^{\text {th }}$ to $27^{\text {th }}$ line: ... . approach, because in the mathematical formulation the positions in between these three distinct points are of no importance.

$34^{\text {th }}$ line: Based on these new ideas, the first author. .

Page xii, 10 th and $11^{\text {th }}$ line: ...the entire spectrum and, for large wave numbers, contains Kraichnan's correct part as a special case.

Page xiii, 33 $^{\text {rd }}$ line: ..."constitutive equation", is reviewed and...

$\mathbf{4 0}^{\text {th }}$ to $\mathbf{4 2}^{\text {nd }}$ line: Many zero-equation turbulence models of Prandtl and his contemporary scientists and engineers are reviewed, and their advantages and disadvantages are discussed.

Page xiv, $\mathbf{1 3}^{\text {th }}$ line: After its development in the middle of the 1980 s and its first publication in ...

$40^{\text {th }}$ line: ... constant in the thermal quasi-equilibrium enstrophy range.

Page $x v, 21$ st $\& 2^{\text {nd }}$ line: ... their care and positive influence on our work-life balances, which is...

$\mathbf{2 6}^{\text {th }}$ line: Good support of production was also given by Shivakumar Krithika.

Page xviii and page 188: 9.2.1 Jets in Quiescent Surroundings

and page 220: 9.2.2 Jets in Parallel Co-flows

Pages xix and 338: Justification of the Quadratic Form of the Energy as a Function of Space and Velocity Coordinates

Page xix and page 358: 11.2.1 What is a Critical...

and page 372: 11.2.7 Turbulent Phase Transition with its Two Phases... 
Page $x x$ and page 413: Appendix E: Fourier Transform for $q$-Generalized Energy...

Page xxi: $\bar{a}(\bar{t})$ Mean acceleration of a fluid lump on the axis of a two-dimensional turbulent jet

$\overline{\mathbf{A}}_{2}$ Averaged second Rivlin-Ericksen tensor

Page xxii: $b$ Half-width of turbulent shear flows, e.g., axi-symmetric jets

$c$ Constant in characteristic function of Lévy flight distribution

Page xxiii: $d a$ Differential surface element

$d v$ Differential volume element

${ }_{L} \mathrm{D}_{+}^{\alpha}$ Left-handed Liouville fractional derivative of order $\alpha$

${ }_{L} \mathrm{D}_{ \pm}^{\alpha}$ Left- and right-handed Liouville fractional derivative of order $\alpha$

$\hat{\tilde{e}}(k)$ Energy density in Fourier space of $\check{e}$ at wave number $k$

$E_{\epsilon}$ Turbulent kinetic energy of a two-dimensional flow

$E_{H}$ Turbulent kinetic energy of a three-dimensional flow

$\check{E}_{\epsilon}$ Generalized energy of a two-dimensional flow

$\hat{\check{E}}_{\varepsilon}(k)$ Fourier transform of the generalized energy $\check{E}_{\epsilon}$

$\check{E}_{H}$ Generalized energy of a three-dimensional flow

Page xxiv: $g\left(x, x^{\prime}\right)$ Nonlocality function, response function

$g_{H}\left(x, x^{\prime}\right) \quad$ Hamba's response function

$g_{K}\left(x, x^{\prime}\right) \quad$ Kraichnan's response function

$G_{21} \quad$ Shear modulus of plane shear flows

Page xxv: $\mathbf{H}$ Hessian tensor of the pressure field

$k-l$ Two-equation turbulence model for the transport of turbulent kinetic energy $k$ and Kolmogorov length $l$

$k-\omega$ Two-equation turbulence model for the transport of turbulent kinetic energy $k$ and vorticity $\omega$

$k$ ' Lowest occurring wave number modulus

$k$ " Highest occurring wave number modulus

K Bachelor's diffusivity tensor ("coefficient")

Page xxvi: $m_{p}$ Scalar magnetization

$m_{0}$ Mass flux of axi-symmetric turbulent jet in the orifice

$n_{\min } \quad$ Eddy density of the eddies on the lower side of $x_{2}^{*}$; see Fig. 7.5 
$n_{\max }$ Eddy density of the eddies on the upper side of $x_{2}^{*}$; see Fig. 7.5

Page xxvii: $\bar{p}_{\max } \ldots$ of the smaller scale...

$\vec{p}_{B}$ Momentum of particle B on trajectory $x_{\mathrm{B}}$

$q$ Index to identify Tsallis's thermodynamic quantities

Page xxviii: $s$ Step size in a Lévy flight distribution

$\widehat{\mathrm{s}}_{ \pm} \quad$ Positive and negative shift operator

Page xxix: $\bar{t} \ldots$ the orifice and moving on the jet axis

$\bar{u}$ Downstream velocity of a fluid lump moving on the axis of a turbulent axisymmetric jet

Page xxx: $\widehat{\vec{u}}_{\vec{n}}^{\left(\kappa^{\prime}, \kappa^{\prime \prime}\right)} \quad \ldots$ and highest wave number $\vec{k}^{\prime \prime}=\kappa^{\prime \prime}\left(n_{1}, n_{2}, n_{3}\right)$

$\widehat{\vec{u}}_{\vec{k}}$ Fourier. .

Page xxxi: $\bar{x}$ Downstream position of a fluid lump moving on the axis of a turbulent axisymmetric jet

$x_{0} \quad$ Core length of a turbulent cylindrical jet

$\bar{x}_{0}$ Streamwise position of a fluid lump moving on the axis of a turbulent axisymmetric jet at the end of the theoretical core

$\bar{x}_{1}$ Streamwise position of a fluid lump moving on the axis of a turbulent axisymmetric jet

Page xxxii : $\alpha$ Order of Liouville fractional derivative

$\alpha$ Exponent of a power law describing the workman's sizes

$\alpha$ Critical exponent for a quantity that approaches criticality from above

$\alpha^{\prime}$ Critical exponent for a quantity that approaches criticality from below

$\beta$ Factor for normal turbulence intensity.

$\beta^{*}$ Exponent. . . to $\mathrm{Re}_{\text {crit }}$

$\gamma$ Critical exponent for a quantity that approaches criticality from above

$\gamma$ Reduction factor for normal turbulence intensities...

$\gamma$ Scale factor in orthotropic turbulence intensity para..

Page xxxiii: $\gamma^{\prime}$ Critical exponent for a quantity that approaches criticality from below $\epsilon_{m}$ Eddy viscosity of turbulent momentum transfer

Page xxxiv : $v$ Critical exponent for a quantity that approaches criticality from above 
$v^{\prime}$ Critical exponent for a quantity that approaches criticality from below

$\xi$ Integration variable in Liouville fractional derivative

Page xxxv: $\sigma_{q}$ Standard deviation of $q$-Gaussian distribution

$\tau_{0}$ Viscous shear stress of flow at the wall

Page 3, ${ }^{\text {th }}$ line: ... boundary, as it was derived with Prandtl's mixing-length...

$\mathbf{1 1}^{\text {th }}$ line: ... working lifetime prior to 1988 , was the most important...

$12^{\text {th }}$ line: Turbulence, by its field quantities, ...

$14^{\text {th }}$ and $15^{\text {th }}$ line: Chaos and turbulence, in all its facets, fascinates...

Page 7: Works of additional scientists and engineers, who studied fluid flows theoretically, numerically or at laboratory scale and contributed significantly to the field of turbulence, will be cited below.

Page 8: Clay,: http. . p prize-problems (2000)

Page 16, $17^{\text {th }}$ line: The two terms describing the divergence of $\left\langle g^{\prime} \vec{u}^{\prime}\right\rangle$ and of $\left\langle\vec{g}^{\prime} \otimes \vec{u}^{\prime}\right\rangle \ldots$

Page $17,1^{\text {th }}$ line: ....contains the substantial derivative. .

Page $18,10^{\text {th }}$ line: Therefore, six unknown components of this tensor must....

Page 19, 17 ${ }^{\text {th }}$ line: $\langle$ mom. eq. $\rangle \cdot\langle\vec{u}\rangle=0 \neq\langle$ mom. eq. $\cdot \vec{u}\rangle=0 \quad$ (20a-c)

Page 20, $6^{\text {th }}$ line: ... more convenient than Eq. (21), but mathematically...

Page 21, 28 ${ }^{\text {th }}$ line: $\langle$ mom. eq. $\rangle \otimes \ldots(29)$

Page 31, $21^{\text {st }}$ line: 4 . Mass of constituents.

Page 33, 21 ${ }^{\text {st }}$ line: $\tau_{21}=-\rho\left(\overline{\mathbf{T}}_{R}\right)_{21}=\ldots$

Page 35, last line: .... effects, from Eq. (16b) it follows that

Page 37, 3rd line: $\tau_{21}=-\rho$. .

$\mathbf{2 3}^{\text {rd }}$ line: ... interpreted as a derivative somewhere between. . .

Page 38, last line: . . . boundary regions, because there the eddy...

Page 39, $11^{\text {th }}$ line: Turbulence is rotational and, therefore, flows are...

Page $42,16^{\text {th }}$ line: This was already noticed by Taylor, who found...

Page $47,16^{\text {th }}$ line: $\ldots \frac{1}{v_{T^{\prime}}}$

Page $49,5^{\text {th }} \& 6^{\text {th }}$ line: ...Prandtl number is defined as the ratio... 
Page 52, $10^{\text {th }} \& 1^{\text {th }}$ line: ... is applied to linear and nonlinear eddy viscosity models.

Page 54, 20 ${ }^{\text {th }}$ line: $\quad \overline{u_{2}^{\prime} u_{1}^{\prime}} \neq \propto \frac{\mathrm{d} \bar{u}_{1}}{\mathrm{~d} x_{2}}, \quad \alpha<\infty, \quad \operatorname{Re} \rightarrow \infty$

$\mathbf{2 5}^{\text {th }}$ line: ... eg., Eqs. 53a,b,69,71, and 89a,b). It immediately. . .

Page 55, $\mathbf{2 1}^{\text {st }}$ line: 3 . The fallacies of turbulence modeling.

$\mathbf{2 5}^{\text {th }}$ line: ...the role of higher-order moments. . .

$\mathbf{2 6}^{\text {th }}$ line: ... influence of higher-order moments. . .

$\mathbf{2 6}^{\text {th }}$ line: ..., then higher-order turbulence modeling. . .

$27^{\text {th }}$ line: ... .superior to lower-order methods.

$\mathbf{2 8}^{\text {th }}$ line: ..., applied to higher-order moment closures, . . .

Page 56, $8^{\text {th }}$ line: ... 1960's and 1970's, because of the ambition to treat complex geometries, was mainly...

Page 57, $17^{\text {th }}$ line: ... in the Reynolds stresses a higher-order term by products of. .

Page 62: $\left.u^{+}\left(y^{+}\right)\right|_{y^{+}=0}=0$

Page 63, $8^{\text {th }}$ line: ... with decreasing thickness as a function of increasing Reynolds number.

$B=A\left[\log _{e}\left(\frac{4}{A}\right)-1\right], \quad A=\frac{1}{\kappa}$

Page 65: Table 5.3 : ... applying Lie group methods, shows that for turbulent shear flows any function and, thus, any scaling law is admissible.

... dependent coefficients (incomplete similarity) is obtained.

Page 66: Toward the wall, approximately the same laws are valid for plane (plate or channel) and cylindrical (pipe) geometries.

Page 67 : $\lim _{\operatorname{Re} \rightarrow \infty} \frac{\bar{u}_{1}\left(\operatorname{Re}, x_{2}\right)}{U}=1$

Page 68 : ..., using Lie group analysis, he demonstrated that any function is fully admissible.

Page 71: Eisner, F., Reibungswiderstand. ..(1932) (in German)

Page 72: Kevlahan, N.K.R., Farge....

Page $76,3^{\text {th }}$ line: ....trajectory of the real particle, contributing to fluctuations and..

Page 79, $\mathbf{2 3}^{\text {rd }}$ line: . . . length of the overall flow field $L$ and its related turnover time $T$ ?

Page 80, $16^{\text {th }}$ line: A first class of nonlocal models...

$22^{\text {nd }}$ line: ... 
Page 84, $\mathbf{2 0}^{\text {th }} \& \mathbf{2 1}^{\text {st }}$ line: After multiplication of both sides of this equation with $d x_{2}$ and introducing the differences...

Page 91, $2^{\text {nd }}$ line: ... . which is the Hessian tensor of the pressure...

$6^{\text {th }}$ line: ..., it follows from Eq. (213a) that

Page $92,5^{\text {th }}$ line: Note the formal analogy with the one-dimensional version given by Eq. (207).

Page 94, 20 ${ }^{\text {th }}$ line: ... where the $\mathrm{D} / \mathrm{D} t$ is the substantial time derivative. The. .

Page 95, $\mathbf{8}^{\text {th }}$ line : . . . been neglected, because in turbulence...

Page 98, 10 ${ }^{\text {th }}$ line: By integrating Eq. (238), it follows that

Page 99, $1^{\text {st }}$ line: $\widehat{u}_{1 a}-\widehat{u}_{1 b}=-\int_{\tau}^{0} \frac{\partial p}{\partial \widehat{x}}(s) d s+\frac{1}{R e} \int_{\tau}^{0} \Delta \widehat{u}_{1} d s$,

$4^{\text {th }}$ line: ... quantities yields the non-normalized

Page 102, $\mathbf{2 8}^{\text {th }}$ line: ... the local strain rate ( local mean velocity gradient $)$ is...

Page 104: Liepmann, H.W.: Mécanique...

Page $107,5^{\text {th }}$ line: ... will be determined for a wake flow behind a cylinder...

Page 108: At this stage, in April 1985, Egolf was somewhat sceptical to take this particular result for granted and to accept it as a basis for further investigation.

Page 112: $\frac{\mathrm{d} \delta_{21}}{d t}=-\lim _{\substack{\Delta t \rightarrow 0 \\ \Delta x_{2} \rightarrow 0}} \frac{\arctan \left(\frac{\delta x_{1}}{\Delta x_{2}}\right)}{\Delta t}$

Page 117: Throughout this page, the averaged quantities $\overline{\Delta x}_{1}$ and $\overline{\Delta x}_{2}$ must be replaced by $\Delta x_{1}$ and $\Delta x_{2}$.

$$
\frac{\mathrm{d} \delta_{\mathrm{T} 21}}{\mathrm{~d} t}=-\lim _{\substack{\Delta t \rightarrow 0 \\ \Delta x_{2} \rightarrow \max }} \frac{\arctan \left(\frac{\delta \bar{x}_{1}}{\Delta x_{2}}\right)}{\Delta t}
$$

Because the fluid motion fluctuates, the average deformation $\delta \bar{x}_{1}$ of a fluid element is considered.

Page 117, $19^{\text {th }}$ line: ... spatial difference $\Delta x_{2}$ and the mean velocity difference. .

Page 121, 16 ${ }^{\text {th }}$ line: $\bar{u}_{2}^{*}=$ const $\times \bar{u}_{1}^{*}$.

Page 125, Table 7.1, ninth line, second row: Density of molecules $n$

Table 7.1, ninth line, third row: Distribution of eddy density $n$

Page 126, $2^{\text {nd }}$ line: Consider a random walk with steps of. . . 
Page 129, $21^{\text {st }}$ line: In the static picture, describing. . .

$22^{\text {nd }}$ line: ... describing probability distributions, we used...

Page 130, $2^{\text {nd }}$ line: ... such as turbulent momentum or particle concentration, are also ...

Page 135: The size of eddies of this class is defined by Kolmogorov's dissipation length scale (see Sect. 7.2.4.11).

Page 141: The special case of Kolmogorov/Obukhov, for infinite Reynolds number flow, $(a=b=2, d=D=3)$ leads to

Page 144: $\widehat{p}_{n}=\rho q_{0}\left(\frac{a}{b^{(2+d-D) / 3}}\right)^{n} u_{0}\left(\frac{1}{b^{n}}\right)^{(1+D-d) / 3}=\ldots$

Page 151: $\quad \kappa_{k, \infty}=\frac{\widehat{P}_{k, \infty}}{\widehat{P}_{0}}=\ldots, \quad \kappa_{m, \infty}=\frac{\widehat{P}_{m, \infty}}{\widehat{P}_{0}}=\ldots$

Page 152, $8^{\text {th }}$ line: This is the DQTM which — at the time of its derivation in 1985was developed by different reasoning.

Page 154, 20 ${ }^{\text {th }}$ line: ... gorov microscales vanish.

Page 160, $6^{\text {th }}$ line: $\ldots$ and consider the Liouville weight $\omega(\lambda, \tau)=\tau^{\lambda-1}(0<\lambda<1)$,

Page 163-165: By replacing the two derivatives in Prandtl's mixing-length model (Eq. 53b) by the left- and right-handed Liouville fractional derivative each, ...

In equations (468b), (470), (473) and (474) the spatial derivatives are the left- and right-handed Liouville fractional derivative ${ }_{L} D_{ \pm}^{\alpha}$.

Page 165, second last line: ... leads to the same result, makes it. . .

Pages 165, 167 and 168: In equations (477), (481) and (482) the derivatives are $\frac{\partial \bar{u}_{1}}{\partial x_{2}{ }^{\prime}}$.

Pages 168, $\mathbf{8}^{\text {th }}$ line: We thus have proven that the DQTM....

Page 171: Einstein, A.: Zur. .Ann. Phys. 19, 371 (1906). (in German)

Page 172: Osterwalder, K.: Methoden. . Mathematics and Physics (1980). . .

Page 175, last line: . . . and the dimensionless Reynolds shear stress and the normal turbulence intensities are

Page 179: $\quad \bar{u}_{1}=U_{G}-\bar{u}_{1}^{*}, \quad \bar{u}_{1}^{*} \geq 0, \quad \bar{u}_{2}^{*}=\bar{u}_{2}$, $(500 \mathrm{a}-\mathrm{c})$

Page 181, 11th line: $\frac{\partial}{\partial x_{1}}\left(\frac{\bar{u}_{1}^{*}}{U_{G}}\right)-\frac{\partial}{\partial x_{2}}\left(\frac{\overline{u_{2}^{\prime} u_{1}^{\prime}}}{U_{G}^{2}}\right)=0$

Page 182, $17^{\text {th }}$ line: Inserting Eqs. (491a) and...

Page 186: ... to obtain with $\sigma=\mathrm{d} b / \mathrm{d} x_{1}$ and Eq. (500a), . .

Page 186, $2^{\text {nd }}$ line: By substituting (522a,d, and e), it follows that 
18 $^{\text {th }}$ line: ... to obtain, with $\sigma=\mathrm{d} b / \mathrm{d} x_{1}$ and Eq. (500a), ...

Page 190: ..., where a decomposition of the downstream velocity $\bar{u}_{1}$ into a horizontal flow...

Page 191, $\mathbf{1}^{\text {st }}$ line: $p_{1} f_{1}-p_{0} \eta \frac{d f_{1}}{d \eta}+\frac{1}{\beta \eta} \frac{d\left(\eta f_{2}\right)}{d \eta}=0$

Page 192, $12^{\text {th }}$ line: The Reynolds shear stress decreases inversely...

Page 192: With Eqs. (554a-d) the momentum equation (549) is simplified to the ODE

Page 200, $15^{\text {th }}$ line: ... propositions:

Page $210,19^{\text {th }}$ line: ...relation, which is stated as:

Page 216, figure 9.20: c) d)

Page 217, $6^{\text {th }}$ line: ... $(647 \mathrm{a}-\mathrm{c})$

$11^{\text {th }}$ line: ...

Page 222, $\mathbf{8}^{\text {th }}$ line: Next, it must be proven that. .

Page 224, $5^{\text {th }}$ line: $f_{21}=\ldots$

Page 226, $13^{\text {th }}$ line: ... turbulence, the DQTM, was derived, contains....

Page 234: Applying these two equations to (720) and dividing the resulting equation by $u^{* 2}$ yields

Page 237, $2^{\text {nd }}$ line: $\ldots=\sqrt{\frac{\beta}{4-\beta}}$,

Page 240: Table 9.3: $\quad g_{1}=1 / 2\langle 1+\sqrt{(1-\chi / \chi)} \tan \{\arctan [\sqrt{\chi /(1-\chi)}] \eta\}\rangle$

Page 248, Fig. Capt.: The flow was calculated with a LES dynamic. . .

Page 249, $9^{\text {th }}$ line $: \ldots$ Reynolds shear stress, $\tau_{21}$,

10 ${ }^{\text {th }}$ line: $\tau_{\text {tot }}=\tau_{0}+\tau_{21}=\mu \frac{d \bar{u}_{1}}{x_{2}}-\rho \overline{u_{2}^{\prime} u_{1}^{\prime}}$.

Page 251, 12 ${ }^{\text {th }}$ line: $\tau_{21}=\ldots$

$15^{\text {th }}$ line: In terms of the self-similar coordinate ...

Page 258, $2^{\text {nd }}$ last line: ... in earlier equations) ${ }^{\prime}:=\mathrm{d} / \mathrm{d} \psi$, lead to ...

Page 260, $4^{\text {th }}$ line: In Eqs. $(727 \mathrm{a}, \mathrm{b})$, replacing $f_{1}(1)$ by $f_{1}(0)$ and substituting these two equations into (844a), it follows that

Page 270, 20 ${ }^{\text {th }}$ line: ...olds numbers up to $35,259,000$, which is the highest $\operatorname{Re}$ value...

Page 271, 23 ${ }^{\text {th }}$ line: .....kind of flows why in this book a deficit power law...

Page 279, Capt. Fig. 9.55: ... . inverse Reynolds number]. 
Page $310,1^{\text {th }}$ line: $\ldots$ $(1015 \mathrm{a}-\mathrm{c})$

Page 310: Replace in Eqs. (1013a,b) $\widehat{u}_{i \vec{n}}$ by $\widehat{u}_{i, \vec{n}}$

Page $311,5^{\text {th }}$ line : ...,

Page 313, $10^{\text {th }}$ line: ...very close to " 1 " (see Sect. 10.4) and power law exponents close to -3 , are expected to occur.

$\mathbf{2 2}^{\text {nd }} \& \mathbf{2 3}^{\text {rd }}$ line: ... of modeling turbulent fields.

Page 322: $\ldots-\lambda_{2}\left[\int_{\Gamma} x^{2} p(x) \mathrm{d} x-\sigma^{2}\right]$,

Page 323: $p(x)=A \exp \left(-\frac{x^{2}}{2 \sigma^{2}}\right)$

Page 325: $\Phi[P]=k \frac{1-\int_{0}^{\infty} P(x) \mathrm{d} x}{q-1}-\ldots$

Page 334, in the figure: ...

Page 335: A comparison of Eq. (1114a) with the maximum value $q$ reveals Eq. (1114c).

Page 343: (a) $E_{\varepsilon}=E$ is the sum of energy contributions $E_{n}: E=\sum_{n=0}^{N} E_{n}$ (see Eq. (1011f))

Page 346: ${ }^{6}$ Temperatures of turbulence were also proposed by Chorin (1994) and Castaign (1996).

Page 347, 13th line: .. and Tsallis et al. (1995)

Page 350: Chorin, A.J., Vorticity and Turbulence. Applied Mathematical Sciences 103. Springer edition, New York, Inc. (1994), ISBN 0-387-94197-5

Page 362: These limit the translational variance for size-scaled eddies to the distance of periodic length $2 \lambda$.

Page $364,3^{\text {th }}$ line: ...

$(1181 \mathrm{a}-\mathrm{c})$

Page $365, \mathbf{2 6}^{\text {th }}$ line: Still a further quantity, that diverges as $s_{1 c}$ is approached, may...

Page 366, $\mathbf{1 8}^{\text {th }}$ line: Following Eq. (1187a-c), the compressibility diverges... $24^{\text {th }}$ line: ...

Page 367, 3rd line: ...

$6^{\text {th }}$ line: ...

$16^{\text {th }}$ line ... $(1197 \mathrm{a}-\mathrm{c})$ 
Page $368,1^{\text {st }}$ line: ...

Page $370,12^{\text {th }}$ line: ...,

Page 376, $19^{\text {th }}$ line: ...

Page 376, $21^{\text {st }}$ line: ...

Page $378,17^{\text {th }}$ line: ...

Page $378,19^{\text {th }}$ line: ...

Page 378, 21 ${ }^{\text {st }}$ line: ...

Page $379,27^{\text {th }}$ line: ...,

Page 383, 8th line: ...

Page 384, 14th line: ...

Page $388,1^{\text {st }}$ line: $\ldots$ domains with disordered and the white with ordered spins.

Page 396, $2^{\text {nd }}$ last line: ... proportion is, which in analogy, we call the marvelous... Page 401: Song, F., Karniadakis,...

Page 413: ...

(D.45a-c)

Page 415: ... (E.7a-c) 